\title{
Imprints of dynamical interactions on brown dwarf pairing statistics and kinematics
}

\author{
M. F. Sterzik ${ }^{1}$ and R. H. Durisen ${ }^{2}$ \\ 1 European Southern Observatory, Casilla 19001, Santiago 19, Chile \\ 2 Department of Astronomy, SW319, Indiana University, Bloomington, Indiana 47405, USA
}

Received 6 December 2002 / Accepted 15 January 2003

\begin{abstract}
We present statistically robust predictions of brown dwarf properties arising from dynamical interactions during their early evolution in small clusters. Our conclusions are based on numerical calculations of the internal cluster dynamics as well as on Monte-Carlo models. Accounting for recent observational constraints on the sub-stellar mass function and initial properties in fragmenting star forming clumps, we derive multiplicity fractions, mass ratios, separation distributions, and velocity dispersions. We compare them with observations of brown dwarfs in the field and in young clusters. Observed brown dwarf companion fractions around $15 \pm 7 \%$ for very low-mass stars as reported recently by Close et al. (2003) are consistent with certain dynamical decay models. A significantly smaller mean separation distribution for brown dwarf binaries than for binaries of late-type stars can be explained by similar specific energy at the time of cluster formation for all cluster masses. Due to their higher velocity dispersions, brown-dwarfs and low-mass single stars will undergo time-dependent spatial segregation from higher-mass stars and multiple systems. This will cause mass functions and binary statistics in star forming regions to vary with the age of the region and the volume sampled.
\end{abstract}

Key words. stars: binaries: general - stars: binaries: visual - stars: formation - stars: low-mass, brown dwarfs

\section{Introduction}

If stars form together in small ensembles, their dynamical evolution is governed by strong, often chaotic gravitational interactions between the cluster members early in their lifetime. This dynamical picture of star formation was advocated several decades ago by Larson (1972) and has recently gained fresh impetus (Larson 2002). Reipurth \& Clarke (2001, hereafter RC) conjecture that brown dwarfs (BD), i.e. star-like objects with masses below the hydrogen burning limit, are actually products of dynamical ejection in fragmenting cloud cores. Due to their ejection, the cores are cut off from their parental reservoir of gas and lose the competitive accretion process to the more massive cores which are not ejected. This scenario is appealing, because a high stellar multiplicity is actually observed in the earliest phases of star formation and because it offers a qualitative explanation for the paucity of BD as close companions. The picture gains theoretical support from recent hydrodynamical star formation calculations where $\mathrm{BD}$ form via fragmentation and collapse of turbulent molecular clouds and are ejected by close triple approaches in unstable multiple systems. The calculations of Bate et al. (2002a) follow collapsing cloud fragments until they reach the opacity limit, implying a minimum fragment mass of about 10 Jupiter-masses $\left(M_{\mathrm{J}}\right)$.

Send offprint requests to: M. Sterzik, e-mail: msterzik@eso.org
Subsequently, these seeds accrete gas from the surrounding envelope and eventually build up to stellar masses. In calculations of this type, Klessen (2001a) and Bonnell et al. (2001) generate a stellar initial mass function (IMF) from turbulent molecular clouds and study dynamical interactions of protostars in a gas-rich environment. However, such simulations do not allow meaningful statistical analysis of the final stellar and BD properties. For instance, in the three-dimensional hydrodynamical star-formation calculations by Bate et al. (2002a), only 50 objects (and only one BD binary) are produced in a total computing time of $\sim 10^{5} \mathrm{CPU}$ hours. Also the numerical scheme employed does not resolve the regions close to the stars, as required for quantitative analysis of the resulting binary orbits.

Our approach is complementary. We calculate many realizations of initially non-hierarchical configurations of fewbody point mass systems by direct orbit integrations, as described in Sterzik \& Durisen (1995, hereafter SD95) and Sterzik \& Durisen (1998, SD98). The initial conditions are motivated by the fact that high resolution maps of molecular clouds reveal a clumpy substructure with a stellar-like mass function. One interpretation is that these are the progenitor clumps in which stars form. These clumps tend to have flattened inner density profiles, and they are likely to fragment into more than one object (Alves et al. 2001). Because the typical dynamical (free-fall) formation time-scale of stellar cores is comparable to the stellar crossing time of the forming cluster, 
the stars will continue to accrete gas when their mutual gravitational forces start to become dynamically important, as discussed in Klessen (2001b). Eventually, however, gravitational interactions will dominate the further stellar dynamical evolution. The combined evolution of small-N clusters incorporating initial gas-rich hydrodynamics and subsequent dynamical interactions has recently been studied by Delgado-Donate et al. (2003). Starting with 5 stellar seeds, they calculate the competitive accretion and dynamical evolution, until (dynamically) stable stellar systems are established. The complexity of the simulations only allows 100 systems to be calculated. They conclude that the statistical analysis of their stellar remnant systems is compatible with current observations regarding mass function, pairing statistics and kinematics.

Our own calculations neglect the effects of remnant molecular gas and disk accretion, but our focus on the stellar dynamical effects alone allow us to compute an extremely large number of cases and enable us to make meaningful and very detailed statistical comparisons with observations. We can then assess whether stellar dynamical processes alone have imprinted themselves on real systems in a verifiable way.

Using this approach, Durisen et al. (2001, hereafter DSP) demonstrate that the observed increase of the multiplicity fraction with stellar primary mass can be understood in terms of dynamical few-body cluster decay, provided that the generation of a stellar initial mass function is a combination of two processes. The total mass of a young cluster is determined by selection from a clump mass spectrum (CMS), while the physics of fragmentation and competitive accretion effectively selects masses for the individual members from a different stellar mass spectrum (SMS) subject to the constraint of the cluster total mass. Because of the range of initial cluster total masses, all stellar masses have a greater chance to be the dominant dynamical mass in some clusters, while still matching a reasonable overall stellar initial mass function. This two-step process does a better job of matching the observed gradual increase in binary fraction with stellar mass than the steep increase found in earlier dynamical decay studies of few-body clusters by McDonald \& Clarke (1993), where cluster members were chosen randomly from an IMF without a total cluster mass spectrum constraint. Dissipation due to gas dynamic effects, as in McDonald \& Clarke (1995), is not required by DSP to match the observational data.

In this paper, we improve upon DSP in several respects. First, we use better initial conditions for our cluster decay calculations. As shown by Sterzik \& Tokovinin (2002), observations of triple systems constrain the likely initial cluster geometry and virial status. The observed alignments of inner and outer orbits is best reproduced by few-body dynamical decay models with a flattened initial geometry and a slight degree of systematic, initial rotation. Second, we include BD's in the SMS using a more realistic BD mass spectrum than DSP. Recent determinations of the sub-stellar mass function tend to support a power-law functional form which can be approximated by $\mathrm{d} N / \mathrm{d} m \propto m^{-\alpha}$ with $\alpha \approx 0.5$ for young open clusters and $\alpha \leq 1$ for the field (Reid et al. 1999; Moraux et al. 2001; Bejar et al. 2001; Chabrier 2002). As we will show, the shape of the IMF in the sub-stellar regime has a significant effect on the pairing statistics for the lowest masses. Here, we will use different observational constraints on the underlying mass function and determine their impact on BD multiplicity and companion fractions.

Our general aim here is to compare predictions with current observations for various assumptions about cluster formation and decay, including our own two-step IMF scenario. Our focus is on the BD mass regime. In Sect. 2, we review our methodology for direct integrations of cluster decays and specify initial conditions. Utilizing the Monte-Carlo pairing scheme introduced by DSP, we then in Sect. 3 compare observed multiplicity fractions with results from various assumptions about dynamical decay. We also derive the fractions of BD secondaries for different primary masses and discuss the differences of the companion and single star mass functions, as well as the mass ratio distributions for BD binaries. Section 4 explores the implications of initial physical scale for the velocity dispersion and binary separation distributions in the BD mass regime. We summarize our results and conclusions in Sect. 5.

\section{Methodology for cluster decay calculations}

First, we describe the initial parameter choices for our "standard $(S)$ " ensemble of cluster decay calculations. We have chosen this as our standard, because, as will become evident, it explains a variety of distinct observations. The numerical orbit integrations are done using the regularization scheme CHAIN of Mikkola \& Aarseth (1993). In contrast to SD95 and SD98, we now integrate 10000 different realizations of initial cluster configurations, rather than just 1000 . In each cluster, the number of cluster members $N$ is allowed to vary such that $1 \leq N \leq 10$.

We follow the DSP two-step approach for assigning masses to cluster members. First, we choose a total cluster system mass $M_{\text {tot }}$ from a CMS $f_{\mathrm{c}}=\mathrm{d} N_{\mathrm{c}} / \mathrm{d} M_{\text {tot }}$, where $N_{\mathrm{c}}$ is the number of clusters. Motivated by Motte et al. (1998), who find that the clump mass spectrum resembles a stellar-like mass spectrum, we use a broken power law $f_{\mathrm{c}} \sim M_{\text {tot }}^{-\alpha_{\mathrm{c}}}$ for the clump mass spectrum, with spectral index $\alpha_{\mathrm{c}}^{l}=0.5$ for $0.1 M_{\odot} \leq M_{\mathrm{tot}} \leq 0.6 M_{\odot}$ and with $\alpha_{\mathrm{c}}^{h}=1.5$ for $0.6 M_{\odot} \leq M_{\mathrm{tot}} \leq 10 M_{\odot}$. Our choices for the clump mass spectral indices are smaller then those of Motte et al. (1998). In fact, our CMS resembles a cloud core mass spectrum for the higher masses rather than a stellar mass spectrum. For the SMS $f_{\mathrm{s}}=\mathrm{d} N_{\mathrm{s}} / \mathrm{d} m$ where $N_{\mathrm{s}}$ is the number of stars, we assume the log-normal distribution in Eq. (3) of DSP, with a characteristic mean $\mu=0.2 M_{\odot}$ and width $\sigma=0.35$ (see also Chabrier 2002; Paresce \& De Marchi 2000 for similar choices of the lognormal parameters). For masses below $0.08 M_{\odot}$, we assume $\mathrm{d} N / \mathrm{d} m \sim m^{-\alpha_{\mathrm{BD}}}$ with $\alpha_{\mathrm{BD}}=0.5$. As the lower mass limit on $\mathrm{BD}$, we use $0.01 M_{\odot}$.

For each cluster in the ensemble, once $M_{\text {tot }}$ is selected, masses are picked randomly from the SMS until the sum is within a tolerance of $\pm 10 \%$ of $M_{\text {tot }}$. If this does not happen by $N=10$, the process starts again for the same $M_{\text {tot }}$ with $N=1$. In this way, we introduce a natural spread for the number of system members without explicitly specifying $N$ or the 


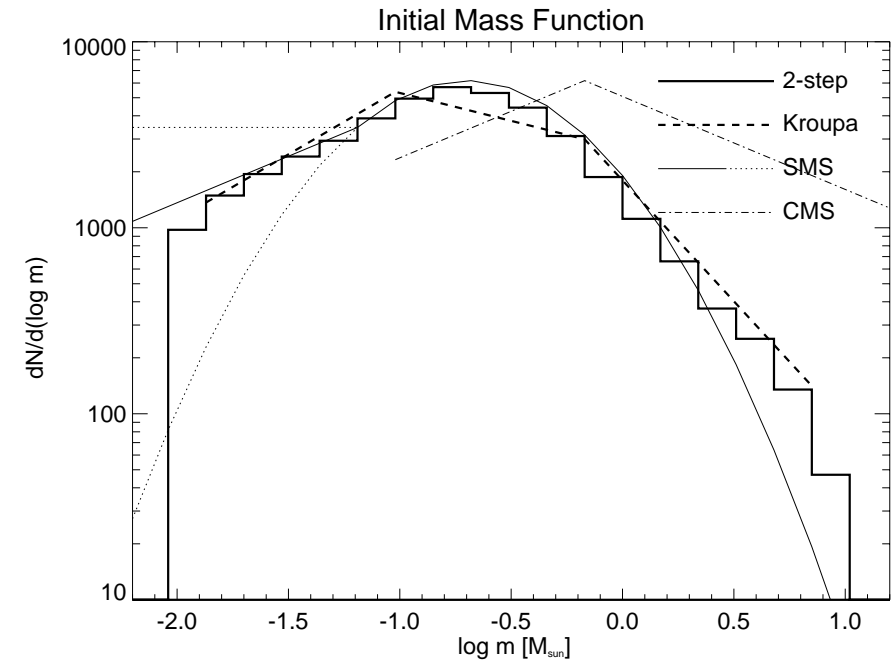

Fig. 1. Mass function used in the simulations (thick histogram), which results from a two-step process with a CMS given by the thin, dasheddotted curve and a SMS given by the thin solid curve for parameter choices referred to as $S$. Alternative choices for the shape of the SMS in the BD regime are dotted, namely a lognormal SMS throughout the $\mathrm{BD}$ regime and a higher abundance of $\mathrm{BD}$ using $\alpha_{\mathrm{BD}}=1$. For comparison, the thick dashed line displays the piecewise power-law IMF based on Kroupa (2001).

distribution of $N$ a priori ${ }^{1}$. These models were designated as "var $N$ " in DSP for "variable $N$ ". In Table 1, we compare the fractions of different initial cluster sizes generated in this way with the ones from DSP (their Table 4) where the BD regime is treated by simply extending the log-normal SMS to sub-stellar masses.

With $\alpha_{\mathrm{BD}}=0.5$, the power-law BD mass function increases the total fraction of BD to $28 \%$ as compared to $17 \%$ in DSP. The lower cluster mass cutoff $\left(0.1 M_{\odot}\right.$ here instead of $0.5 M_{\odot}$ in DSP) now produces a larger fraction of $N=1$ and 2 systems. These single and binary systems obviously do not participate in any dynamical interactions and constitute a "primordial" population that preserves their formation signature without being altered. In this respect, our proposed scenario is much less radical than the one described by RC, in which every object, especially BD, participate in three-body dynamics. Our prescription delivers a natural distribution of cluster sizes, including primordial singles and binaries. We are not aware of any firm argument that excludes single or binary star formation for theoretical or observational reasons. We therefore include these systems in our analysis and allow the possibility that a certain fraction of single and binary systems are actually formed primordially.

Figure 1 displays the generated IMF, indicates the functional forms of the assumed SMS and CMS, and compares our IMF with the galactic field star IMF from Kroupa (2001). Our resultant IMF is consistent with the observed IMF over three decades in $m$. This is not a great surprise, however, because a reasonable IMF fit was one of our criteria for adopting the parameters of our standard model.

${ }^{1}$ Note that our choice of the lower clump mass of $0.1 M_{\odot}$ implies that BD do not form directly from $N=1$ cores.
Later, we will study the influence of variations in the IMF parameters. More BD (38\%) are generated with $\alpha_{\mathrm{BD}}=1$; we refer to this parameter set with a higher $\mathrm{BD}$ content as $H$. A similar amount of BD (37\%) results from $\alpha_{\mathrm{BD}}=0.5$, but assuming CMS power-law indices suggested by Motte et al. (1998), specifically $\alpha_{\mathrm{c}}^{l}=1.5$ and $\alpha_{\mathrm{c}}^{h}=2.5$. We call this parameter set $M$. The $M$ case produces an IMF which has 10 's \% excesses of M-stars and and factor of three to ten deficits for the solar and higher-mass stars relative to the observational IMF. Such differences are probably unacceptable. The $M$ case is included here as an extreme example with a very steep CMS.

The choice of the initial geometry and the virial status of the clusters is derived from an analysis of relative orbit orientations in visual triple systems. In SD95, geometry is specified by choosing positions randomly over a uniform spheroidal distribution of possible positions. Flattening or elongation are specified by the ratios of the axes. Velocities can also be assigned as a combination of random and systematic motions. Initial uniform rotation is parameterized by $\beta$, the ratio of the rotational kinetic energy to absolute value of the total system energy. Sterzik \& Tokovinin (2002) show that, with oblate (10:1) cluster geometries and moderate initial rotation $(\beta=0.1)$, dynamical decay can explain the modest correlation seen in the orbital alignments of real triples. These values only roughly characterize the initial cluster configuration, and a broader distribution is expected for real systems. SD95 have already shown that only extreme choices of geometries or virial states significantly alter the results of our dynamical decay calculations. We therefore believe that the actual choice of these values - if not too extreme - does not change our conclusions.

The $6261 N>2$ clusters in our standard 10000 cluster ensemble are integrated for 300 cluster crossing times $\left(T_{\mathrm{cr}}\right)$ until stable bound sub-systems (or remnants) are formed. Not all meta-stable systems have decayed into a long-term stable final configuration by this time. Semi-empirical stability criteria must be applied to judge their final system state, but only $5 \%$ of all systems are affected by this uncertainty. This will therefore not alter our interpretation significantly.

\section{Results from Monte-Carlo simulations}

Let us now compare three distinct classes of stellar cluster decay models which span a range of plausible alternatives. Rather than computing actual decays for all these cases, we will use the Monte Carlo (MC) methods of DSP to generate large ensembles which are normalized to the direct orbit integration results for the standard case.

1-Step: Bias. It is well known that cluster dynamics tend to favor pairing of objects with large masses, a process sometimes termed "dynamical biasing". Suppose, within a given cluster, we sort the member masses $m_{1}, m_{2}, m_{3}, \ldots$ such $m_{1} \geq$ $m_{2} \geq m_{3} \geq \ldots$. Then, "complete" dynamical biasing means that only the most massive pair $m_{1}, m_{2}$ will form a stable binary, while all other cluster members will be ejected as singles. If the masses for these stars are chosen randomly from an IMF without regard to a cluster mass constraint, we refer to this class of models as "1-step: bias" models. McDonald \& Clarke (1993) studied this scenario analytically but found the resulting 
Table 1. Distribution of sizes $N$ for progenitor clusters in DSP and here. Also given are the relative proportions of various remnant bound system sizes after dynamical decay calculations have been run for 300 crossing times $\left(T_{\mathrm{cr}}\right)$. The numbers in the table are fractions of the total number of bound systems. The 10000 systems of row two have decayed into 26352 systems by row three. The primordial systems with $N=1$ $(N=2)$ contribute a fraction of $7 \%(35 \%)$ to the final number of singles (binaries) after the decay calculations, while practically all initial clusters with $N>2$ decay during $300 T_{\text {cr }}$ and boost the absolute number of singles and binaries.

\begin{tabular}{lcccccccccc}
\hline \hline$N$ & 1 & 2 & 3 & 4 & 5 & 6 & 7 & 8 & 9 & 10 \\
\hline DSP & 0.05 & 0.11 & 0.15 & 0.14 & 0.12 & 0.10 & 0.09 & 0.08 & 0.08 & 0.09 \\
$S$ this work & 0.18 & 0.19 & 0.14 & 0.11 & 0.08 & 0.07 & 0.06 & 0.06 & 0.06 & 0.06 \\
$300 T_{\text {cr }}$ & 0.67 & 0.21 & 0.08 & 0.03 & $2.8(-3)$ & $2.3(-4)$ & $4.6(-3)$ & $\ldots$ & $\ldots$ & $\ldots$ \\
\hline
\end{tabular}

mass-ratio and binary fraction distributions are incompatible with observations. This class of models represents an important fiduciary extreme assumption.

2-Step: Dynamics. As argued in DPS, true weighting factors for the pairing probabilities in a cluster deviate from $100 \%$ dynamical biasing and have to be determined by direct orbit integrations. In addition, with a "2-step" mass selection process via a CMS and an SMS, DSP showed that dissipationless cluster decay dynamics yields mass-ratio and binary fraction distributions which are reasonably consistent with observations. We therefore introduce a second conceivable class of models "2-step: dynamics", in order to reflect the two-step mass selection and the true cluster decay dynamics. For this paper, we determine weighting factors based on the detailed numerical orbit integrations for the conditions described in Sect. 2. Examining the remnant binaries after $300 T_{\mathrm{cr}}$, we find that $P\left(m_{1} m_{2}\right)=0.57, P\left(m_{1} m_{3}\right)=0.31$, and $P\left(m_{2} m_{3}\right)=0.12$, where $P\left(m_{i} m_{j}\right)$ is the probability of forming a binary $m_{i} m_{j}$ from the ordered masses. Pairings with even lower mass stars in the cluster are negligible. Note that complete biasing assumes $P\left(m_{1} m_{2}\right)=1$.

2-Step: Random. McDonald \& Clarke (1995) included star-disk interactions in order to model the effects of gas dynamics on cluster decay and found that the selection of secondary masses in binaries is essentially randomized when including strong dissipation. This also increases the fraction of lower-mass primaries significantly. Recently, Malkov \& Zinnecker (2001) studied the influence of the mass function on binary statistics and mass ratio distributions with MC simulations by assuming all stars are binaries and using random pairing weights. They find a good agreement with observed binary fraction statistics provided brown dwarf secondaries are considered undetectable, but the mass ratio distribution for the lowest mass (M-type) primaries appears to be incorrect. In our implementation, we impose the cluster mass constraint but completely randomize the pairings in each cluster, presumably due to strong dissipative effects during stellar encounters. In practice, what we do is use $P\left(m_{1} m_{2}\right)=1$ before sorting the cluster members by mass. We refer to this third class of model as "2-step: random".

Our MC approach is the same as DSP, but with different sub-stellar mass functions, a broken CMS with a smaller lower mass cut-off, and with updated probabilities for the 2-step: dynamics case. Having $10^{5}$ clusters in the MC computations guarantees statistically reliable results. One binary is generated in each cluster according to the above scenarios, and the pairing statistics are then analysed for the entire ensemble.

\subsection{Multiplicity fractions}

First, we consider the dependence of the multiplicity on the primary mass. Although the MC generates only binaries (and singles), the interpretation of the binary fraction includes higherorder systems in an approximate way, because many of the meta-stable higher-order remnants of orbit integrations will eventually decay into binaries and singles anyhow and because stable multiples are in effect represented in the statistics by the hardest binary in the multiple. Figure 2 displays multiplicity fractions $(M u F)$ versus the primary mass. The data points are, to our knowledge, all observations of $M u F$ for different primary mass ranges, which represent a statistically meaningful sample. We try to estimate fair primary mass ranges and errors from the original references, if they are not directly given. We use the following studies: Reid et al. (2001, R01), Martin et al. (2000, M00), Close et al. (2003, C03), Reid \& Gizis (1997a, R97a), Reid \& Gizis (1997b, R97b), Leinert et al. (1997, L97), Fischer \& Marcy (1992, FM92), Marchal et al. (2002, MDF02), Duquennoy \& Mayor (1991, DM91), Shatsky \& Tokovinin (2002, ST02).

The different curves in Fig. 2 refer to different MC model classes. All are based on the standard parameter set $S$ in the following sense. We first generate clusters by the two-step process described in Sect. 2. The resulting ensemble of stars and $\mathrm{BD}$ represents our reference IMF. The same IMF is guaranteed is all cases by using this same ensemble of stars. For both 2-step classes of model, we use the two-step assignment of member masses to clusters and then form one binary (multiple) from each cluster using the appropriate method, as explained above. For the "1-step: bias" case, we keep the same stars/BD and the same cluster size distribution, but we randomly reassign members to clusters and use complete biasing to create pairs.

The $M u F$ is always strictly 0 for the lowest masses in all three model classes, because, with a lower cutoff in the IMF, no lower mass secondaries can exist. The full line (labelled "2-step: dynamics") refers to the DSP dynamical binary formation model with updated weights and mass function. This model is in reasonably good agreement with observations, especially for the stellar mass range. As already noted by McDonald \& Clarke (1993), the "1-step: bias" case produces an $M u F$ that does not - except for an intermediate mass 


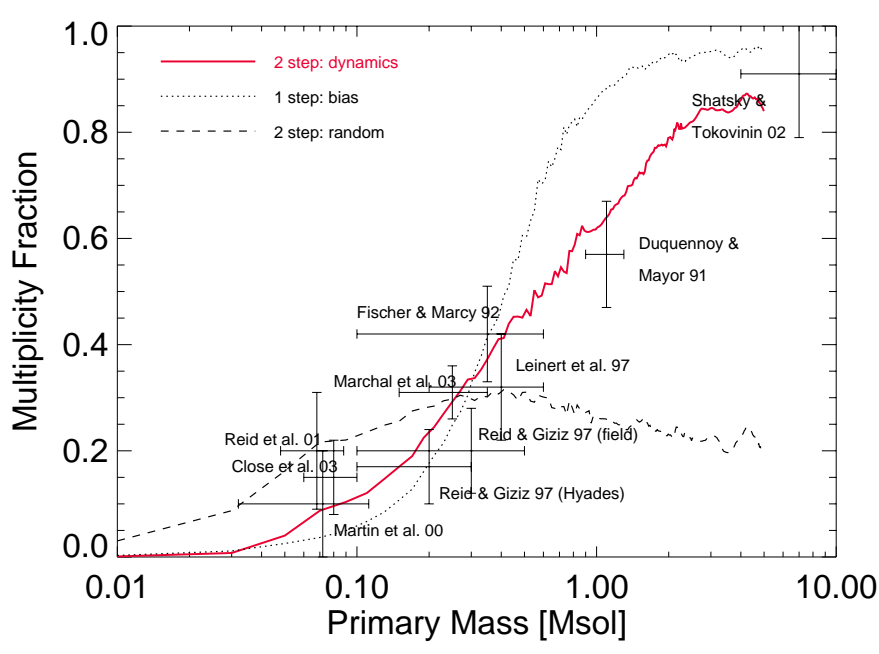

Fig. 2. Multiplicity fractions versus primary mass for different models, compared with observational data.

range - fit the more gradual increase with mass which is actually observed. In the "2-step: random" case, the $M u F$ is relatively smooth. It increases significantly for very low masses to a broad peak near the peak in the IMF, then falls away gently at high masses. This case does not match the data at all for main sequence stars of type earlier than spectral class $\mathrm{K}$. This random pairing approach is very different from that of Malkov \& Zinnecker (2001), where all stars and BD's are paired.

Table 2 summarizes the overall $M u F$ as a function of mass range for the different cluster formation and pairing models. We map spectral types along the main sequence to corresponding mass bins, as in DSP, in order to ease comparisons with observations. The $\mathrm{BD}$ mass regime is divided into $\mathrm{L}$ and $\mathrm{T}$ types with an adopted transition value of $0.05 M_{\odot}$, which is approximately valid for $1 G y r-o l d ~ B D$. The $M u F$ for each class of MC model is generated with three alternative choices of the IMF parameters $(S, H, M)$. For reference, we include the $M u F$ of the DSP model. The final column in Table 2 refers to the observations shown in Fig. 2.

The three main model classes are characterized by the same trend already evidenced in Fig. 2 for all parameter sets $S, H$ and $M$. The "1-step: bias" class is characterized by a rather steep increase of the $M u F$, whereas the "2-step: random" class exhibits a rather flat $M u F$ over all masses. The "2-step: dynamics" class is intermediate. Within one class, the differences of $S, H$ and $M$ are most pronounced for low mass primaries. $H$ cases and, even more so, $M$ cases boost the $M u F$ for BD and $\mathrm{M}$-type stars. In order to guide the eye in Table 2, we emphasize observationally consistent values (within 0.01 of the error bars) in boldface. Obviously, observed $M u F$ within specific mass ranges can be synthesized by different model classes and parameter choices. But the "2-step: dynamics" class with the IMF parameter choices $S$ and $H$ come closest to the observational fractions over two orders of magnitude in primary mass.

We conclude from Table 2 that the $M u F$ for the lowest masses is a sensitive measure of the formation process and the underlying IMF. A high $M u F$ in the sub-stellar regime is favored by random pairing mechanisms and by a high fraction of BD in the initial IMF. A 1-step IMF and/or IMF shapes that have too few objects in the BD mass domain, especially the lognormal distribution in DSP, decrease this fraction and are probably incompatible with current observations of the $M u F$. For the parameter choices ( $S$ and $H$ ) which have acceptable IMF's, dynamical cluster decay based on pure gravitational point-mass interactions yields multiplicity fractions around $10 \%$ in the BD mass regime. More randomized pairing statistics, expected for instance from dissipative encounters, appear to be necessary to boost this fraction significantly. But if pairing is randomized for all masses, the observed increase of the $M u F$ over the stellar mass regime cannot be explained. In the entire stellar mass regime, the "2-step: dynamics" class of model appears to be a very good description of the observed $M u F$. Latest studies having high statistical significance suggest a $M u F$ of $15 \pm 7 \%$ for primary masses around the stellar/sub-stellar boundary (Close et al. 2003). This is in full accord with the $8-18 \% M u F$ we obtain from the "2-step: dynamics" model with standard parameter assumptions at comparable mass ranges. However, if the observed BD $M u F$ turns out to be as high as $20 \%$, our calculations suggest that dissipation is relatively more important for the production of BD pairs than for stellar pairs. As a cautionary remark for any comparison with observations, the primary mass ranges considered have to be assessed very carefully, because of the expected strong dependence of the $M u F$ on the primary mass.

\subsection{Brown dwarf companion frequencies}

The MC calculations also reveal the fraction of BD secondaries per primary mass bin. In Table 3, we summarize the results from the three main classes of MC models. Again, for each class, we demonstrate the influence on BD companion frequency of the parameter set choices $S, H$, and $M$. L and Tdwarfs are lumped together in this table, and we define the BD companion fraction as

$M u F_{\mathrm{BD}}(P)=\frac{N\left(B_{\mathrm{BD}} \mid P\right)}{N(S \mid P)+N(B \mid P)}$

where $N\left(B_{\mathrm{BD}} \mid P\right)$ is the number of binary systems that have a companion in the BD mass regime and a primary in mass bin $P$ and where $N(S \mid P)$ and $N(B \mid P)$ are the number of single and binary systems having a (primary) mass $P$, respectively. This definition guarantees that the total $M u F$ for primary mass $P$ is the sum of the companion frequencies for all secondary mass bins.

As evidenced by Table 3, BD are rare companions to stars, as generally expected from interaction dynamics (MC95). As far as we are aware, however, this is the first time that anyone has quantitatively compared the influence of different physical processes and mass functions on the predicted companion frequencies. The "2-step: dynamics" model predicts a gradual decrease of the BD companion frequency from late M-type primaries to early type stars. Assuming the standard $S$ set of parameters, $9 \%$ of all stars with masses corresponding to a late $\mathrm{M}$ spectral type should have BD companions, whereas BD companions are expected for only $2 \%$ of stars more massive then $1.2 M_{\odot}$. With an enhanced BD fraction, as in $H$, the BD 
Table 2. Multiplicity fractions as function of mass range calibrated in spectral types on the main sequence (for stars). Three classes of models are considered: 2-step dynamics (2sd), 1-step: bias, 2-step random, and 2-step: dynamics. Subclasses codes $S, H$ and $M$ are explained in the text.

\begin{tabular}{lcccccccccccc}
\hline \hline spectral & mass & \multicolumn{1}{c}{ 1-step: bias } & \multicolumn{3}{c}{ 2-step: random } & \multicolumn{1}{c}{ 2-step: dynamics } & observations \\
type & range & $S$ & $H$ & $M$ & $S$ & $H$ & $M$ & $S$ & $H$ & $M$ & DSP & \\
\hline $\mathrm{T}$ & $0.01-0.05$ & .01 & .02 & .05 & .08 & .11 & .21 & .01 & .01 & .07 & 0 & \\
$\mathrm{~L}$ & $0.05-0.08$ & .03 & .05 & $\mathbf{. 1 5}$ & $\mathbf{. 2 1}$ & $\mathbf{. 2 4}$ & .48 & $\mathbf{. 0 8}$ & $\mathbf{. 1 0}$ & .40 & $2.6(-4)$ & $.20 \pm .11(\mathrm{R} 01), .10 \pm .10(\mathrm{M} 00)$ \\
$\mathrm{M}-$ & $0.08-0.27$ & $\mathbf{. 1 2}$ & $\mathbf{. 1 5}$ & .41 & $\mathbf{. 2 6}$ & $\mathbf{. 2 7}$ & .35 & $\mathbf{. 1 8}$ & $\mathbf{. 2 1}$ & .36 & .05 & $.17 \pm .07, .20 \pm .08(\mathrm{R} 97 \mathrm{a}, \mathrm{b}), .15 \pm .07(\mathrm{C} 03)$ \\
$\mathrm{M}+$ & $0.27-0.47$ & $\mathbf{. 4 1}$ & $\mathbf{. 4 5}$ & .73 & $\mathbf{. 3 0}$ & $\mathbf{. 2 9}$ & $\mathbf{. 4 0}$ & $\mathbf{. 3 8}$ & $\mathbf{. 4 0}$ & .55 & $\mathbf{. 3 5}$ & $.32 \pm .10(\mathrm{~L} 97), .42 \pm .09$ (FM92), .31 $\pm .05(\mathrm{MDF} 02)$ \\
$\mathrm{K}$ & $0.47-0.84$ & .71 & .71 & .80 & .29 & .27 & .36 & .51 & .53 & .62 & .51 & \\
$\mathrm{G}$ & $0.84-1.20$ & .86 & .88 & .81 & .27 & .26 & .33 & $\mathbf{. 6 3}$ & $\mathbf{. 6 4}$ & $\mathbf{. 7 1}$ & $\mathbf{. 6 7}$ & $.58 \pm .10(\mathrm{DM} 91)$ \\
$\mathrm{F}+$ & $>1.20$ & $\mathbf{. 9 3}$ & $\mathbf{. 9 5}$ & $\mathbf{. 8 1}$ & .23 & .23 & .27 & $\mathbf{. 7 8}$ & $\mathbf{. 7 9}$ & $\mathbf{. 8 0}$ & $\mathbf{. 8 7}$ & $.91 \pm .12(\mathrm{ST} 02)$ \\
\hline
\end{tabular}

Table 3. BD companion frequencies, including both $\mathrm{L}$ and $\mathrm{T}$ types, as a function of spectral type of the primary. The BD row includes only L-type primaries. The number in parentheses indicates the companion frequency in the lowest mass bin $(\mathrm{T})$ only. The MC models and corresponding parameter sets are explained in the text.

\begin{tabular}{lcccccccccc}
\hline \hline & \multicolumn{3}{c}{ 1-step: bias } & \multicolumn{3}{c}{ 2-step: random } & \multicolumn{4}{c}{ 2-step: dynamics } \\
Primary & $S$ & $H$ & $M$ & $S$ & $H$ & $M$ & $S$ & $H$ & $M$ & DSP \\
\hline BD & $.03(.02)$ & $.05(.04)$ & $.15(.11)$ & $.21(.17)$ & $.24(.21)$ & $.48(.41)$ & $.08(.06)$ & $.10(.08)$ & $.40(.33)$ \\
M- & $.05(.03)$ & $.07(.04)$ & $.18(.10)$ & $.17(.12)$ & $.20(.16)$ & $.25(.18)$ & $.09(.05)$ & $.11(.08)$ & $.23(.15)$ & $1.6(-3)$ \\
$\mathrm{M}+$ & $.05(.03)$ & $.07(.05)$ & $.15(.09)$ & $.13(.09)$ & $.15(.12)$ & $.19(.13)$ & $.07(.04)$ & $.10(.06)$ & $.17(.10)$ & .02 \\
$\mathrm{~K}$ & $.05(.03)$ & $.07(.05)$ & $.13(.08)$ & $.11(.07)$ & $.13(.10)$ & $.14(.10)$ & $.06(.03)$ & $.09(.05)$ & $.12(.07)$ & .03 \\
$\mathrm{G}$ & $.05(.03)$ & $.06(.04)$ & $.13(.08)$ & $.08(.06)$ & $.11(.08)$ & $.11(.07)$ & $.03(.02)$ & $.05(.03)$ & $.08(.04)$ & .02 \\
$\mathrm{~F}+$ & $.04(.02)$ & $.05(.03)$ & $.11(.06)$ & $.06(.04)$ & $.09(.07)$ & $.08(.06)$ & $.02(.01)$ & $.03(.02)$ & $.03(.01)$ & .01 \\
\hline
\end{tabular}

companion frequencies are slightly higher, and the $M$ set of parameters roughly doubles the BD companion frequency. If random pairing processes dominate the formation of $\mathrm{BD}$ companions, as a high BD $M u F$ might suggest, the BD companion fractions are dramatically enhanced, especially for higher-mass primaries. The "1-step: bias" cases all have an almost uniform BD companion fraction for all primary masses, corroborating the analytic results of McDonald \& Clarke (1993). This fraction is $\sim 5 \%$ for $S$, increases to $\sim 7 \%$ for $H$, and is $\sim 14 \%$ for the $M$ case parameters.

Note that, for the "2-step: random" class of models, the BD $M u F$ for F+ stars should be roughly equal to the total $M u F$ of the $\mathrm{F}+$ stars times the fraction of BD in the IMF. It is easy to verify that this is the case in the table. The tendency for the BD companion fractions to increase toward lower mass for "2-step: random" is a direct reflection that BD's are a greater fraction of the possible pool of secondaries as the primary mass decreases. Non-random pairing and cluster decay dynamics tend to lower the fractions of BD secondaries substantially.

The values given in Table 3 provide another useful basis for comparisons with observations. Considerable effort has been undertaken in the past few years to determine BD companion frequencies to stars. The first BD, Gl 229B, was actually found as a visual companion of an M 1 star by Nakajima et al. (1995), and this provoked intensive searches by various research groups. Meanwhile, the so-called "brown dwarf desert", a strong deficit of BD companions to stars with periods below one year, now appears to be well established for solar-type stars (Halbwachs et al. 2000). Modern precision radial velocity measurements designed to detect planets do not reveal objects of BD mass as close companions, although they could be easily detected with these techniques. A firm limit on the fraction of close $\mathrm{BD}$ companions for such orbit periods is less then $1 \%$.

For larger separations, the situation is less clear. Direct imaging searches demand high contrast and high angular resolution observing techniques, and the detection sensitivity is a strong function of angular separation. The companion's mass, if detected, depends on a reliable age determination and theoretical evolutionary models and is therefore uncertain, especially for young objects. No statistically complete study probing the separation regime between 1 and $30 \mathrm{AU}$ with sufficient sensitivity is currently available. So it is not possible to derive BD companion fractions over the entire possible separation range. All observed companion frequencies are therefore only lower limits to the true companion frequency. In their analysis of optical and NIR coronagraphic observations of a sample of 107 nearby $(<8 \mathrm{pc})$ stellar systems, Oppenheimer et al. (2001) derive a BD companion fraction of $1 \%$ having masses $>40 M_{\mathrm{J}}$ and ages $<5 \mathrm{Gyr}$ in a separation range ranging from 40 to $120 \mathrm{AU}$. Their sample is approximately complete to M 5 spectral types and is dominated by $\mathrm{K}$ and early M-type stars. Their result is consistent with an earlier study by Reid \& Gizis (1997b). Even smaller separations and a higher contrast were probed using WFPC2 onboard HST by directly imaging 23 stars within 13 pc of the Sun (Schroeder et al. 2000). No BD companion was found. For G, K, \& M-stars, McCarthy (2001) claims a BD companion fraction of $1 \pm 1 \%$ orbiting between 75 and 300 AU based on 102 objects imaged with the KECK infrared coronagraph, while Lowrance (2001), utilizing NICMOS coronagraphy, finds a slightly higher BD fraction in a sample of 50 younger stars 


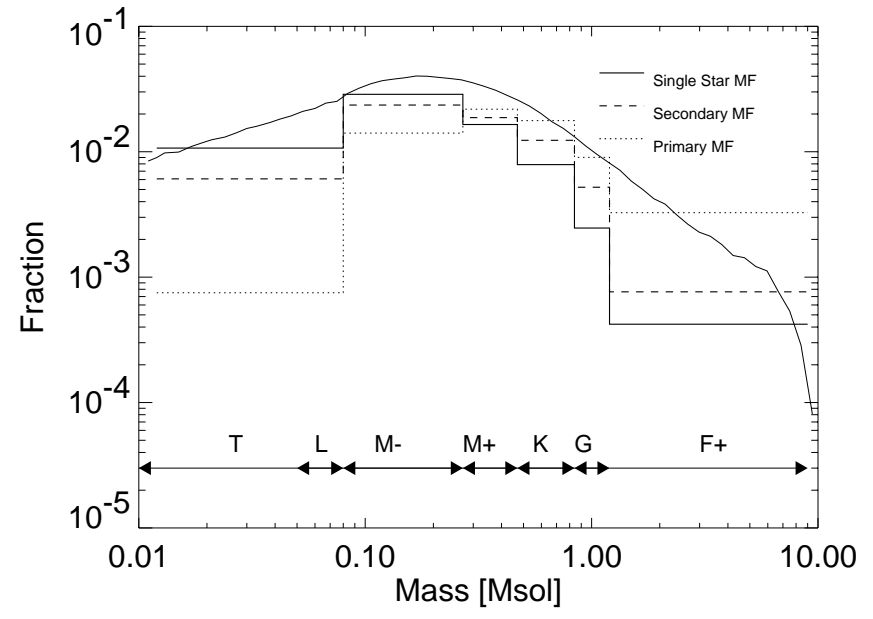

Fig. 3. Comparison of primary, secondary, and single star mass functions for the "2-step: dynamics model".

located in the TW Hya and Tucanae associations. It is too early to draw final conclusions on the BD companion frequencies and their dependence on the primary mass. Based on current observations, BD companion fractions for solar-type stars probably do not exceed a few percent for separations $<1 \mathrm{AU}$ and $>30 \mathrm{AU}$. Future observations will have to address this issue and should be able to discriminate the proposed binary formation models by examining the dependence of BD companion fraction on primary mass.

We conclude that both the gradual increase of $M u F$ for stars over a wide mass range and the relatively low BD companion fractions found for stars are in general agreement with the results of our "2-step: dynamics" model, in which the IMF is generated by parameters set $S$. We therefore base the remaining analyses on this model.

\subsection{Mass ratio distributions}

Few-body dynamics, if a dominant agent in the early evolution of stellar systems, imprints specific differences on the shapes of the mass functions for singles stars and for the primaries and secondaries of binary systems. Kroupa (2001) demonstrates in numerical simulations of stellar clusters that $N$-body dynamics affect the mass function and introduce biases in observational determinations due to unresolved binaries. Our MC simulations permit us to determine such biases as well. In Fig. 3 we assume the 2-step: dynamics model with the standard choice of IMF parameters $S$ and plot the underlying IMF (full line, same as in Fig. 1), i.e., the mass function that is constructed when each star in a stellar system is counted individually. The histogram is constructed to match the mass-spectral type relation introduced above. The heavy solid histogram encompasses only single stars, while the dashed (dotted) line counts only the secondary (primary) components in a binary system. The relative proportions of each object class varies greatly across the mass spectrum. While BD binaries are rare with respect to single BD by a factor of 12 , one finds early type stars $\sim 9$ times more often as a primary component in a binary then as a single object. This is - in principle - an observable signature, and should be revealed in careful analysis of number counts

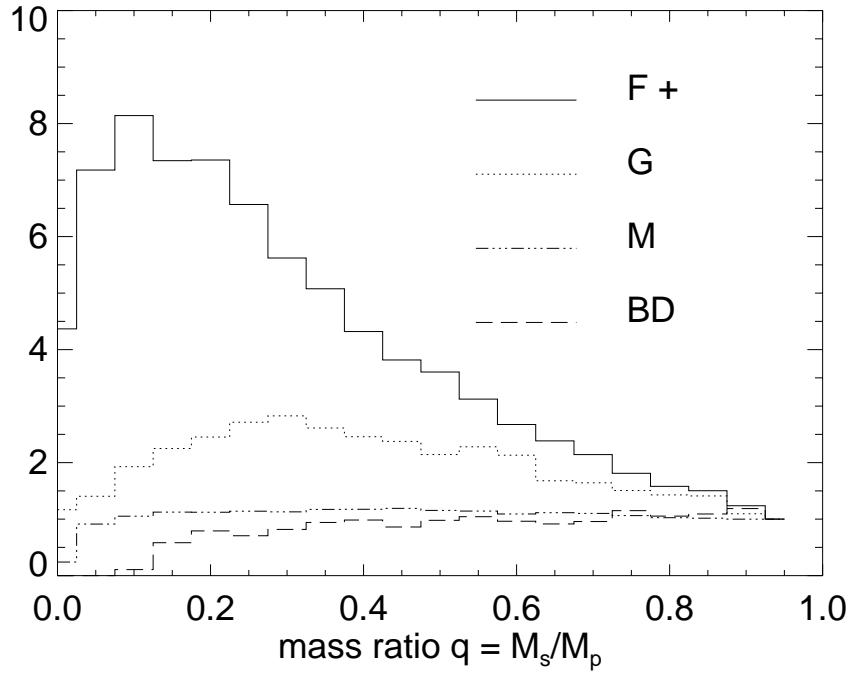

Fig. 4. Mass ratio distribution for selected primary mass types for the "2-step: dynamics model".

in the solar neighborhood. Moreover, we find that companion mass functions produced by the "2-step: dynamics model" are rather independent of the primary mass, as already shown rigorously for the "1-step: bias model" in MC93. We therefore expect most probable companion masses around $0.2 M_{\odot}$ (see also Fig. 3).

More specific predictions can be made with respect to mass-ratio distribution in binaries. For selected mass-spectral type bins, we show the distribution of the mass ratio $q=$ $M_{\mathrm{s}} / M_{\mathrm{p}}$ in Fig. 4 , where $M_{\mathrm{s}}$ and $M_{\mathrm{p}}$ are the secondary and primary masses, respectively. It has already been noted before that dynamical decay models can match the $q$-distributions for solar-type stars (MC93, Valtonen 1997; DSP). For very lowmass stars and BDs, the $q$-distribution is distinctly different from that for G-stars. Our model produces $q$-distributions for low-mass primaries which are almost flat and even slightly rising toward $q=1$. Observationally, Reid \& Gizis (1997b) in their analysis of low-mass binaries in the solar neighborhood note that the mass-ratio distribution is inconsistent with the assumption that both components are drawn from the same IMF independently; instead equal mass binaries are favored.

\section{Results from direct orbit integrations}

We will now discuss properties of dynamical decay remnants which can only be determined by direct orbit integrations. Velocity dispersions and the separation distribution of forming binaries fall into this category, as both result from the same dynamical process, a close triple approach with energy exchange. The analytic functional forms of the distributions for both separation and velocity distributions of decay remnants from clusters with a variable mass spectrum were derived and then verified by direct integrations in SD98. From partial energy equipartition during an encounter, lower-mass objects receive higher recoil velocities. On the other hand, binding energies induced by encounters will result in closer separations for lower-mass components. In the following, we quantify these behaviors. 


\subsection{System scales}

Some statistical properties in decaying clusters can be understood in terms of scale-free quantities, such as the multiplicity fractions and mass ratio distributions discussed in earlier sections. Physical scales need to be introduced for determining velocities and separations. Observations of the typical stellar spacings in early stages of star formation now approach the required angular resolution to resolve multiple protostars. Apparently, many cloud cores can be resolved into common envelope multiple systems, with typical spacings of a few 100's of AU (see Looney 2000). On the theoretical side, fragmentation calculations such as Bate et al. (2002a) or Boss (2002) are just reaching the necessary resolution to derive typical separations of stellar fragments, before few-body dynamical processes take over their further evolution.

Our 2-step mass selection process prescribes how many objects $N$ form in a given cluster. Its mass is drawn from a CMS and therefore not a free parameter. But the system scale size is not defined a priori. SD95 related the initial scale length of dynamical decaying clusters with typical conditions expected in fragmenting cloud cores and clumps. As reference, they used a $3 M_{\odot}$ stellar cluster with a typical "virial radius" of $R_{H}=125 \mathrm{AU}$. SD98 later demonstrated that the assumed mass versus size relation (or, equivalently, the specific energy versus mass relation) is critical for determining how dispersion velocities and binary separations depend on mass.

Let us now assume that the precursor clumps of the fewbody stellar systems have radial density profiles that resemble a Bonnor-Ebert type structure. Then the clump mass versus size relation is of a simple linear form $M_{\mathrm{c}} \propto R_{\mathrm{c}}$, with $M_{\mathrm{c}}, R_{\mathrm{c}}$ being the clump mass and size. This relation is indeed the one observed by Motte et al. (1998) and Motte et al. (2001) and implies that the specific energy in star forming clumps is constant over the observed mass and size ranges. How do we relate this observation to the stellar cluster properties? It seems reasonable to assume that the total clump mass and the total stellar masses are related via a (relatively) constant star formation efficiency. Unfortunately, a simple mapping from clump scale to an initial cluster scale is not yet available. For the following discussion, we assume that the specific energy of the initial stellar clusters is also roughly constant. This follows if the initial clump size $R_{\mathrm{c}}$ and the cluster size are linearly related, e.g. if the clumps retain a fixed number (i.e, $N$ ) Jeans masses as they evolve to a cluster configuration (a similar assumption is made in Delgado-Donate et al. 2003). Constant specific energy is equivalent to constant virial speed $v_{\text {vir }}$ of the few-body clusters. As in SD98, we choose $v_{\text {vir }}=3.3 \mathrm{~km} \mathrm{~s}^{-1}$ for all clusters. In reality, we expect some dispersion about this value. As long as the distribution of $v_{\text {vir }}$ for clusters of all mass peaks at about the same value, the arguments in the section below remain valid.

\subsection{Velocity distributions}

In Fig. 5, we present various cumulative speed distributions for BD from our numerical calculations. The dotted line indicates single $\mathrm{BD}$, whereas the dashed line represents speeds of the center of mass of BD as primaries in BD pairs and the dashed-dotted line refers to system speeds having BD as secondary companions to stars. For comparison, the distributions for single "solar"-type stars (K, G, F and earlier types) and binaries with solar-type primaries are plotted as the heavy solid and thin solid lines, respectively. The left-hand asymptotes of these cumulative distributions indicate that a finite fraction of $\mathrm{BD}$ and stars have extremely low or zero velocities. These come mostly from our $N=1$ or $N=2$ systems that do not undergo dynamical interactions.

A mass-velocity dependence for singles is not large, but obvious from Fig. 5. The K, G, F singles have median speeds of about $1 \mathrm{~km} \mathrm{~s}^{-1}$, while single BDs have a median speed of about $2 \mathrm{~km} \mathrm{~s}^{-1}$. High velocity $\left(v>5 \mathrm{~km} \mathrm{~s}^{-1}\right)$ escaping single stars (dubbed "run-away TTS" in Sterzik et al. 1995) exist but are rare. Delgado-Donate et al. (2003), including hydrodynamical effects, find similar speed distributions for their sample of ejected single stars, although they do not notice a significant variation with mass. The influence of the remnant cloud potential on ejection speeds needs still to be addressed in future, high statistics, studies. The binary systems containing one BD (dotdash and dashed curves) have a much lower dispersions than other systems. This is partly because about half of all binaries with at least one BD component stem from $N=2$ clusters and so get no kick velocity from the dynamics. Of course, in reality, all clusters and decay remnants would share the random motions of the cloud clumps from which they formed; so the observed velocity distributions would be a convolution of the cloud clump velocity distribution with the velocity distributions resulting from cluster decay. In total, $90 \%$ of all binaries with at least one BD companion gain only small (or zero) extra velocity kicks $\left(<1 \mathrm{~km} \mathrm{~s}^{-1}\right)$ from dynamic decay. We expect only small differences in the speed distributions for different primary masses of these BD-containing binaries, and a discrimination of these dispersion velocities by direct observations of radial velocities or proper motions would be difficult, especially when the clump velocity dispersion is folded in. The single BD do have higher speeds, but $90 \%$ of them are $<5 \mathrm{~km} \mathrm{~s}^{-1}$. It is not so surprising that the measured radial velocities of a sample of $9 \mathrm{BD}$ in the Chameleon SFR do not reveal evidence for run-away objects in the study by Jörgens \& Günther (2001).

As shown in Fig. 5, the differences between the speed distributions for single stars and binary or multiple star systems are large enough that characteristic observable signatures are expected in the kinematics. Sterzik et al. (2001) presented a crude model for the spatial and temporal evolution of a loose association where BD, stars of different mass, binaries, and multiples emanate from a hypothetical "star forming volume" with velocity distributions resulting from cluster decay. The typical velocity dispersion of a few $\mathrm{pc} / \mathrm{Myr}$ for single BD is sufficient to generate spatially extended BD halos, and to deplete $\mathrm{BD}$ in the sites of active star formation within a few Myr after their formation. On the other hand, binaries and multiple systems are expected to stay close to their sites of formation much longer due to their lower intrinsic velocity dispersion. A spatial gradient in the IMF and $M u F$ should grow with time in such a scenario. Observationally, indications exist that loose young stellar associations like Taurus-Auriga indeed have low BD surface densities (Luhman 2000) and a high $M u F$. However, 


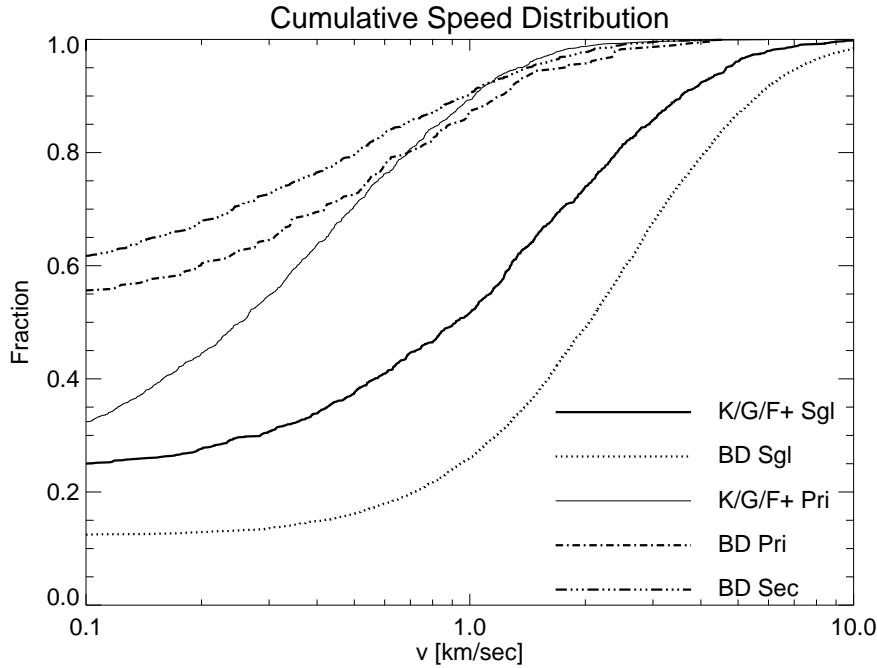

Fig. 5. Cumulative velocity dispersion distribution for different masses for the "2-step: dynamics model".

more sophisticated models are required which includes effects of the remnant cloud and of dynamical interactions in dense environments (e.g. the Trapezium cluster; Kroupa et al. 1999) before quantitative comparisons of different star forming regions and open clusters can be made.

\subsection{Binary separations}

Separation distributions are a key to understand the formation and angular momentum history of binaries. But the prototypical separation distribution published by Duquennoy \& Mayor (1991) for solar-type stars, which is characterized by a broad log-normal distribution function centered around $30 \mathrm{AU}$, still awaits theoretical explanation. Larson (2002) favors stochastic, dynamical processes as a dominant agent in broadening the separations. Kroupa \& Burkert (2001), on the other hand, demonstrate that the observed broadness can never be obtained by dynamical evolution in dense stellar clusters alone. We have shown in SD98 that an initially narrow mean stellar separation distribution in small $N$ clusters can be broadened by a factor 5-10 (see also Bonnell 2001). This is helpful but not enough to explain the extreme breadth of the observed binary separation distribution. Additional physical processes such as tidal friction, dissipation due to disks, and a distribution of initial conditions probably must be taken into account. Observers even find hints of a temporal evolution of the $M u F$ for young stars (Patience et al. 1998) and of a possible formation site dependence (Brandner \& Köhler 1998). The separation distribution is also likely to depend on primary mass.

Few-body cluster decay models allow us to model the expected separation distribution. In SD98 we already noted a trend for systems with lower mass primaries to have smaller separations when the initial cluster specific energy is constant for different clusters. In Fig. 6 we present the results for the current model in the form of cumulative separation distributions, grouped by different primary masses. The number of systems per primary mass bin is given in the legend. The distribution functions are in general roughly Gaussian, with a tail towards

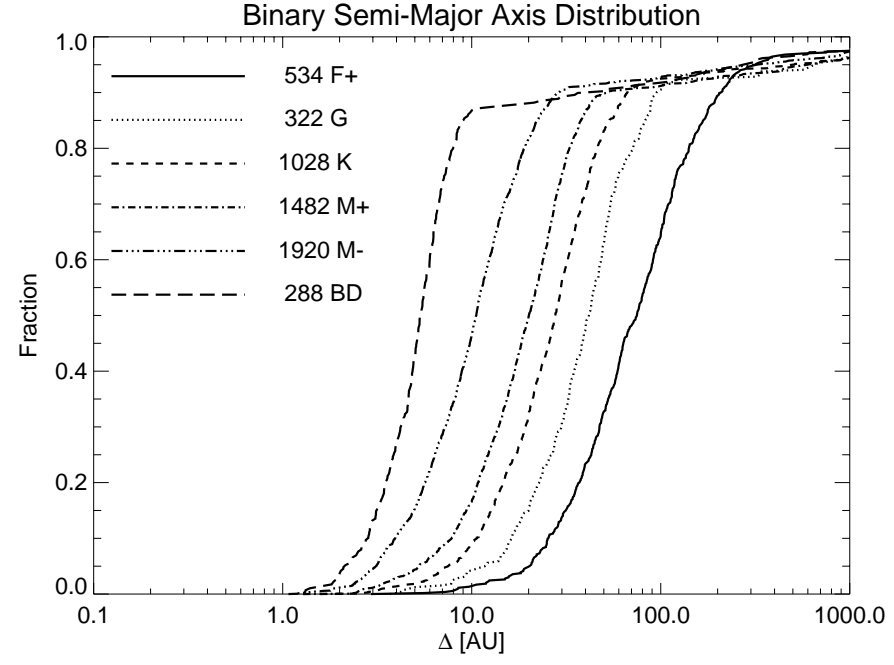

Fig. 6. Cumulative binary separation distribution for different primary masses for the "2-step: dynamics model".

larger separations. Binaries with smaller masses have smaller separations, due to two effects. (1) In a three-body encounter, the kinetic energy of the escaper equals the gain in binding energy for the remaining binary. If this binary is of low mass, the binding energy increase causes more hardening of the orbit then for a higher mass system. Moreover, low-mass binaries only survive energetic encounters if they are close systems. (2) If the specific energy of the clusters is the same, the system scale is proportional to the total cluster mass. This implies that low mass systems are already initially in a more compact cluster configuration; the subsequent dynamical interactions only enhance this trend.

Quantitatively, our choice of physical scale reproduces the peak of the G-type binary separation distribution at about $30 \mathrm{AU}$. The lowest mass binaries produced in the simulation, 288 BD pairs, have median separations around $4 \mathrm{AU}$. This is in remarkable agreement with available observations. All confirmed binary BD systems to date all have separations below 15 AU, a value not much affected by selection bias (Martin \& Basri 2001). The most recent separation distribution analysis of very low-mass binaries by Close et al. (2003) corroborates the much smaller peak of their semi-major axis distribution. With similar high statistical significance than in the BD case, a careful analysis of the binary properties of a nearby M-star sample indicates that their mean separations are significantly lower then for the G-stars but higher then the BD binaries (Marchal et al. 2002).

\subsection{Miscellaneous}

In this section we explore additional BD properties in the framework of dynamical interaction processes in young fewbody clusters. Most of the observational data now available have even lower statistical reliability then those discussed above, but they will eventually provide important clues about details of the BD formation mechanisms. 


\subsubsection{Brown dwarfs in higher-order multiples}

The triple and higher-order systems produced in our decay calculations add up to about $11 \%$ of all configurations (cf. Table 1). Some of these systems, especially those with high eccentricities, and/or small period or mass ratios, are only metastable and will decay further on in their evolution. About one-third of all triples contain at least one BD and fully $10 \%$ contain two BD. This is a non-trivial number. Multiple BD companions to stars should be found at the percent level. The preferred configuration is a close BD companion to a primary with not too extreme mass ratio. The third BD companion is found in an outer, hierarchical orbit. Typical distances are about ten to a hundred times larger then those of the inner semi-major axis. According to our calculation, a minority (about 10\%) of two-BD cases in triples have a configuration where a close BD binary orbits a more massive primary. It is interesting to note that at least one of these configurations has been found, namely, the close (semi-major axis $\approx 1$ AU) BD binary GJ569B, orbiting an M2 type primary at a distance of 50 AU (Martin et al. 2000). The three systems in our standard $S$ calculation that resemble such a configuration indeed have comparable axis and mass ratios. After applying the same physical scaling discussed above, two BD binary separations are around $2 \mathrm{AU}$, whereas the wider, stellar, component is between 30 and $110 \mathrm{AU}$. The third system is a very wide triple, with 750 and $5200 \mathrm{AU}$ as inner and outer semi-major axis. So two of our three systems match the observed system parameters surprisingly well.

Genuine BD triple systems, i.e. systems composed entirely of $\mathrm{BD}$, are rare in the simulations and make up only about $0.2 \%$ (i.e. 34 of 2177 total) of all triple systems in our standard case. Observations will sooner or later find dynamically stable BD multiples, despite their rarity, because there should be a strong detection bias in magnitude-limited surveys.

\subsubsection{Wide BD companions}

Contrary to the established BD desert at very narrow separations ( $<3 \mathrm{AU})$, and the apparent scarcity of BD companions in the intermediate separation range (10 AU to $1000 \mathrm{AU}), \mathrm{L}$ and T-dwarf common proper motion companions to $\mathrm{M}$ to $\mathrm{F}$ mainsequence stars with separations $>1000$ AU seem to be comparatively common (Gizis et al. 2001). Their mass ratios are generally low $(q<0.15)$, in contrast to the high mass ratios typically found for the few close BD companions.

Figure 7 illustrates the dependence of the mass ratio on separation for different types of BD companions based on our calculations. With a few exceptions, BD binaries (small filled circles) have narrow separations, typically below 10 AU. Their mass ratios are evenly distributed, with a lower cutoff defined by our IMF. BD as secondaries to stars (large open circles) tend to have similar separation distributions, but preferentially populate the lower mass ratio regime. A significant number of low- $q$ binaries can therefore be formed by dynamical decay, but note, that $2 / 3$ of them stem from primordial $N=2$ binaries. A few low-mass ratio binary systems have large separations. BDs as components of a triple systems are plotted as large, filled circles. They populate a distinct area, having large

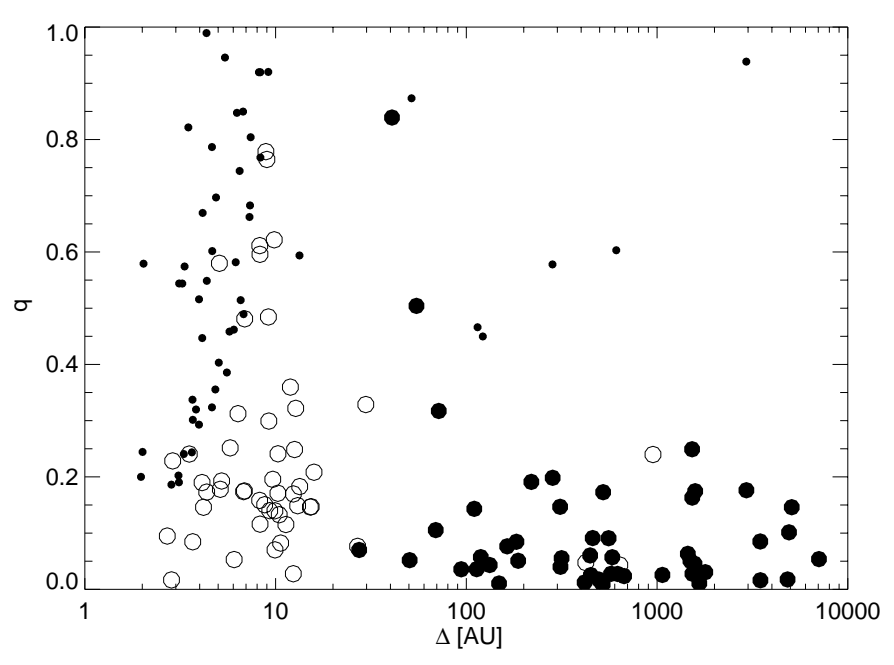

Fig. 7. Mass ratio $q$ versus separation $\Delta$ distribution for various BD secondary populations for the "2-step: dynamics model". Binary BD: small filled circles; BD companions to stars: large open circle; BD companions in triples: large filled circles.

separations and small mass ratios. Reid et al. (2001) present a similar graph. Their 15 real systems exhibit a bimodal distribution: close, high- $q$ BD binaries and wide, low- $q$ BD companions to stars or stellar systems. Unlike our Fig. 7, the low- $q$, small separation region is not populated in Reid et al. One obvious reason for this would be observational bias against the discovery of close $(\Delta<10 \mathrm{AU})$, low-mass ratio companions to higher-mass stars because of blending. In the pure dynamical model presented here, these systems should be present. If our model parameters are right, we would expect that the stellar primaries of observed wide, low- $q$ BD's will mostly turn out to be binaries or higher-order systems, re-inforcing a similar prediction by Delgado-Donate et al. (2003).

\subsubsection{Very close BD binaries}

Although BD companions to solar-type stars are almost absent for separations $<1 \mathrm{AU}$, at least one spectroscopic BD binary pair exists, PP115 (Basri \& Martin 1999). Its orbital solution yields a semi-major axis of $\Delta=0.03 \mathrm{AU}$. The formation of spectroscopic stellar binaries is still enigmatic (see e.g. the discussion in Tohline \& Durisen 2001), and it is clear also from our calculations that dynamical interactions alone cannot account for these close binaries, neither in the stellar nor in the BD mass regime. It is interesting to note that spectroscopic binaries tend to occur more often in hierarchical systems (Tokovinin \& Smekhov 2002). One way to shrink an inner orbit is through a Kozai effect due to the presence of a third body (Kiseleva et al. 1998). The required perpendicular orbit configuration occurs frequently in decaying few-body clusters (Sterzik \& Tokovinin 2002), but PPI15 apparently is not accompanied by a third low-mass system member. Its origin is puzzling.

Recently, Bate et al. (2002b) claim the formation of close binary systems by a combination of dynamical interactions and orbital decay alone, but the separations achieved by this 
process are 1 to 2 orders of magnitude wider then in spectroscopic binaries.

\subsubsection{BD disks}

If the same formation mechanism of low-mass pre-main sequence stars extends to objects in the brown dwarf mass range, accretion disks should be a common byproduct of BD formation. Searches for signatures of circumstellar disks around young BDs are ongoing. Muench et al. (2001) find that more then half of the population of substellar objects in the Trapezium cluster exhibit excess near infrared emission and interpret this as evidence that these objects are surrounded by circumstellar disks similar to those for the stellar population of the cluster. NIR observations trace - in principle - the innermost regions of disks, but current evolutionary models for very young brown dwarfs are too uncertain to infer reliable NIR photospheric models. The mid-infrared region appears to be a more reliable probe of excess emission, and Natta \& Testi (2001) demonstrate that the measured MIR excesses in a few bona fide $\mathrm{BD}$ in the Chameleon region are in fact well described by models of circumstellar disks identical to those associated with T Tauri stars, scaled down to keep the ratio of the disk-to-star mass constant. Natta \& Testi (2001) conclude that there is a similar formation mechanism for TTS and $\mathrm{BD}$, though they caution that even the MIR data only constrain disk parameters for sizes less than $\sim 1$ AU. Detection of much larger silhouette disks around BD might provide another useful constraint on the birth environment of very low-mass objects. If dynamical interactions are important in the early lifetime of BD, as in the BD ejection formation scenario from $\mathrm{RC}$, the typical disk truncation radius will roughly be of the order of the closest periastron passage (Hall et al. 1996), which is around 5-10 AU, the typical BD binary separation obtained in this work. We conclude that material at radii $<5-10$ AU should be unaffected by the encounter process. Only observations at longer wavelengths are sensitive enough to constrain the outer disk radii of $\mathrm{BD}$.

$\mathrm{BD}$ formation as a result of ejection during an unfinished accretion process in stellar clusters implies that all BD have been formed in this way, as in the model of RC. We note a specific difference in the relative importance of this process in our own scenario. If the progenitor clump mass spectrum extends below the sub-stellar limit, then BD could also form alone down to a mass set by the fragmentation limit (Low \& Lynden-Bell 1976; Padoan et al. 1997). In the two-step scenario outlined above we assumed a lower CMS cutoff of $0.1 M_{\odot}$. In our prescription for picking $N$ masses, this cutoff was not low enough to produce single BDs directly (but with the assumed CMS and SMS, $55 \%$ of all binary BDs and $59 \%$ of all binaries having one $\mathrm{BD}$ companion are actually primordial, i.e. directly formed from $N=2$ "clusters"). If the lower CMS cutoff mass is in the BD regime, a high fraction of BD will directly condense out of very-low mass clump fragments and not participate in further cluster dynamics. Such objects could have extensive disks. We do not attempt to estimate this fraction because the lower cutoff of the CMS is simply not known.

\section{Conclusions}

In this contribution we have presented a framework for understanding how brown dwarf properties may result from dynamical interactions in young clusters. Our picture assumes that stars and brown dwarfs condense from progenitor clumps in a hierarchical fragmentation picture. With choices of a clump mass spectrum, and a stellar mass spectrum, a simple, statistical, two-step process produces an IMF that is compatible with current observations. The distribution of the number of objects $N$ in the few-body clusters is a natural side-product. Taking into account the dynamical evolution of the clusters with $N>2$, we draw quantitative and statistically significant conclusions about the expected multiplicity fraction, the secondary mass function, the mass ratio distribution, and the binary separation distribution of brown dwarfs.

Our essential results are as follows:

1. Our standard model is in good agreement with observed slowly rising multiplicity fraction for primary masses ranging from about $0.1 M_{\odot}$ to $10 M_{\odot}$. In the brown dwarf regime, the expected $M u F$ is somewhat dependent on the shape of the IMF. Observed brown dwarf companion fractions around $15 \%$ for very low-mass stars as reported recently are not inconsistent with dynamical decay models. If observed BD binaries are as frequent as $20 \%$, however, they are difficult to explain by dynamical processes alone. Dynamical evolution including dissipation and/or a higher fraction of primordial BD pairs appear to be necessary to boost the $M u F$ in the lowest mass regime. However, dissipation and random pairing cannot account for the multiplicity statistics of solar-type stars. Perhaps young BDs are more affected by dissipation during encounters then stars.

2. Dynamical interaction processes in the early evolution of young clusters harboring brown dwarfs imprint a distinct secondary (or companion) mass function on binaries and higher-order multiples. BD are expected to be rare companions to stars ( $\leq 5 \%$ for solar-types), and BD companions should be found more frequently around low-mass stars. This prediction is in general agreement with current observations, although much higher statistical significance is required to discriminate alternative assumptions about the IMF and the pairing statistics.

3. The mass ratio distributions for BD binaries and low-mass stars are expected to be rather flat, compared with distributions that peak at low mass ratio for higher-mass primaries. The same trend is observed.

4. $\mathrm{BD}$ dispersion velocities are of the order of $2 \mathrm{~km} \mathrm{~s}^{-1}$. This is somewhat higher but not dramatically different from stellar dispersion velocities. Nevertheless, it will lead to a timedependent spatial segregation of single BDs and multiple stellar systems in star forming regions. Current observations remain inconclusive on this point.

5. With the additional assumption of constant specific energy for our initial clusters, the proposed model yields a median of the binary separation distribution that is in full agreement with current observations from the BD to solar-type mass regime. 
Acknowledgements. We would like to thank C. Clarke, R. Larson, and $\mathrm{H}$. Zinnecker for their comments on an earlier version of the manuscript. The referee, Cathie Clarke, helped to further improve and clarify this paper. R.H.D. was supported in this research by NASA Grant NAG5-11964.

\section{References}

Alves, J. F., Lada, C. J., \& Lada, E. A. 2001, Nature, 409, 159

Bate, M. R., Bonnell, I. A., \& Bromm, V., 2002a, MNRAS, 332, L65

Bate, M. R., Bonnell, I. A., \& Bromm, V., 2002b, MNRAS, 336, 705

Basri, G., \& Martin, E. 1999, AJ, 118, 2460

Bejar, V. J. S., Martin, E. L., Zapatero Osorio, M. R., et al. 2001, ApJ, 556,830

Bodenheimer, P. 1978, ApJ, 224, 988

Bodenheimer, P., \& Burkert, A. 2001, in Birth and Evolution of Binary Stars, ed. H. Zinnecker, \& R. D. Mathieu (San Francisco: ASP), Proc. IAU Symp., 200, 13

Bonnell, I., \& Bate, M. R. 1994, MNRAS, 269, L45

Bonnell, I. A. 2001, in Birth and Evolution of Binary Stars, ed. H. Zinnecker, \& R. D. Mathieu (San Francisco: ASP), Proc. IAU Symp., 200, 23

Bonnell, I. A., Bate, M. R., Clarke, C. J., \& Pringle, J. E. 2001, MNRAS, 323, 785

Boss, A. 2002, ApJ, 568, 743

Brandner, W., \& Köhler, R. 1998, ApJ, 499, L79

Chabrier, G. 2002, ApJ, 567, 304

Close, L. M., Siegler, N., Freed, M., \& Biller, B. 2003, ApJ, in press

Delgado-Donate, E. J., Clarke, C. J., \& Bate, M. R. 2003, MNRAS, submitted

Durisen, R. H., Sterzik, M. F., \& Pickett, B. K. 2001, A\&A, 371, 952 (DSP)

Duquennoy, A., \& Mayor, M. 1991, A\&A, 248, 485 (DM)

Fischer, D. A., \& Marcy, G. W. 1992, ApJ, 396, 178 (FM)

Gizis, J. E., Kirkpatrick, J. D., Burgasser, A., et al. 2001, ApJ, 551, L163

Hall, S. M., Clarke, C. J., \& Pringle, J. E. 1996, MNRAS, 278, 303

Halbwachs, J. L., Arenou, F., Mayor, M., Udry, S., \& Queloz, D. 2000, A\&A, 355, 581

Jörgens, V., \& Günther, E. 2001, A\&A, 379, L9

Kiseleva, L. G., Eggelton, P. P., \& Mikkola, S. 1998, MNRAS, 300, 292

Klessen, R. 2001, ApJ, 556, 837

Klessen, R. 2001, ApJ, 550, L77

Kroupa, P. 2001, MNRAS, 322, 231

Kroupa, P., \& Burkert, A. 2001, ApJ, 555, 945

Kroupa, P., Petr, M., \& McCaughrean, M. 1999, New Astron., 4, 495

Larson, R. B. 1972, MNRAS, 156, 437

Larson, R. B. 2002, MNRAS, 332, 155

Leinert, C., Henry, T., Glindemann, A., \& McCarthy, D. W., Jr. 1997, A\&A, 325, L159

Looney, L. 2000, ApJ, 529, 477

Low, C., \& Lynden-Bell, D. 1976, MNRAS, 176, 367

Lowrance, P. J. 2001, Ph.D. Thesis, University of California, Los Angeles

Luhman, K. L. 2000, ApJ, 544, 1044
Malkov, O., \& Zinnecker, H. 2001, MNRAS, 321, 149

Marchal, L., Delfosse, X., Forveille, T., et al. 2002, in Brown Dwarfs, ed. E. Martin, Proc. IAU Symp. 211, ASP, in press

Mardling, R. A., \& Aarseth, S. J. 2001, MNRAS, 321, 398

Martin, E. L., Brandner, W., Bouvier, J., et al. 2000, ApJ, 543, 299 (M00)

Martin, E. L., Koresko, C. D., Kulkarni, S. R., et al. 2000, ApJ, 529, L37

Martin, E. L., \& Basri, G. 2001, in Birth and Evolution of Binary Stars, ed. H. Zinnecker, \& R. D. Mathieu (San Francisco: ASP), Proc. IAU Symp., 200, 55

Mazeh, T., Latham, D. W., Goldberg, E., et al. 2001, MNRAS, 325, 343

McCarthy, D. 2001, Ph.D. Thesis, University of California, Los Angeles

McDonald, J. M., \& Clarke, C. J. 1993, MNRAS, 262, 800

McDonald, J. M., \& Clarke, C. J. 1995, MNRAS, 275, 671

Mikkola, S., \& Aarseth, S. J. 1993, Cel. Mech. Dyn. Astr., 57, 439

Moraux, E., Bouvier, J., \& Stauffer, J. R. 2001, A\&A, 367, 211

Motte, F., Andre, P., \& Neri, R. 1998, A\&A, 336, 150

Motte, F., André, P., Ward-Thompson, D., \& Bontemps, S. 2001, A\&A, 372, 41

Muench, A. A., Alves, J., Lada, C. J., \& Lada, E. A. 2001, ApJ, 558, L51

Nakajima, T., Oppenheimer, B. R., Kulkarni, S. R., et al. 1995, Nature, 378,463

Natta, A., \& Testi, L. 2001, A\&A, 376, L22

Oppenheimer, B. R., Golimowski, D. A., Kulkarni, S. R., et al. 2001, AJ, 121, 2189

Padoan, P., Nordlund, A., \& Jones, B. J. T. 1997, MNRAS, 288, 145

Paresce, F., \& De Marchi, G. 2000, ApJ, 534, 890

Patience, J., Ghez, A. M., Reid, I. N., Weinberger, A. J., \& Matthews, K. 1998, AJ, 115, 1972

Reid, I. N., Kirkpatrick, J. D., Liebert, J., et al. 1999, ApJ, 521, 613

Reid, I. N., Gizis, J. E., Kirkpatrick, J. D., \& Koerner, D. W. 2001, AJ, 121, 489 (R01)

Reid, I. N., \& Gizis, J. E. 1997a, AJ, 114, 1992 (R97a)

Reid, I. N., \& Gizis, J. E. 1997b, AJ, 113, 2246 (R97b)

Reipurth, B., \& Clarke, C. 2001, AJ, 122, 432 (RC)

Schroeder, D. J., Golimowski, D. A., Brukardt, R. A., et al. 2000, AJ, 119,906

Shatsky, N., \& Tokovinin, A. 2002, A\&A, 382, 92 (ST02)

Sterzik, M. F., Alcala, J. M., Neuhäuser, R., \& Schmitt, J. H. M. M. 1995, A\&A, 297, 418

Sterzik, M. F., \& Durisen, R. H. 1995, A\&A, 304, L9 (SD95)

Sterzik, M. F., \& Durisen, R. H. 1998, A\&A, 339, 95 (SD98)

Sterzik, M. F., Durisen, R. H., \& Pickett, B. K. 2001, in Young Stars Near Earth, ed. R. Jayawardhana, \& T. Greene (San Francisco), ASP Conf. Ser., 244, 116

Sterzik, M. F., \& Tokovinin, A. A. 2002, A\&A, 384, 1030

Tokovinin, A. A., \& Smekhov, M. G. 2002, A\&A, 382, 118

Tohline, J. E., \& Durisen, R. H. 2001, in Birth and Evolution of Binary Stars, ed. H. Zinnecker, \& R. D. Mathieu (San Francisco: ASP), Proc. IAU Symp., 200, 40

Valtonen, M. J. 1997, ApJ, 485, 785 